\title{
$M$-SEMIREGULAR SUBALGEBRAS IN HYPERFINITE FACTORS
}

\author{
BY \\ SISTER RITA JEAN TAUER( $\left.{ }^{1}\right)$
}

1. Introduction. The general study of algebras of operators on Hilbert space has led to the investigation of rings of operators, also called $W^{*}$-algebras or von Neumann algebras. If the center of a ring (center in the algebraic sense) consists only of scalar multiples of the identity, then the ring is a factor. Since every ring can be decomposed into factors [6], the study of rings is, in a sense, reduced to a study of factors. In this paper we are concerned with the maximal abelian subalgebras of type $\mathrm{II}_{1}$ factors, or continuous factors which have a finite trace defined on them [2]. For the present, we restrict ourselves to the study of hyperfinite factors, that is, those which are generated by a sequence of factors $\mathfrak{M}_{n}$ of type $I_{n}$, with $\mathfrak{M}_{n_{1}} \varsubsetneqq \mathfrak{M}_{n_{2}} \varsubsetneqq \cdots$. (The factor $\mathfrak{M}_{n}$ is isomorphic to the algebra of $n$ by $n$ matrices.) Since all hyperfinite factors are algebraically isomorphic [5, \$4.7], while the concept of a subring of a finite factor is purely algebraic [5, \$1.6], any construction used will yield general results.

Dixmier has defined three types of maximal abelian subalgebras $\boldsymbol{R}$ in a factor $\mathfrak{A}$ : regular, semiregular, and singular [3]. These depend on the properties of $N(\boldsymbol{R})$, the ring generated by $\left\{V: V \boldsymbol{R} V^{*}=\boldsymbol{R}, V\right.$ unitary, $\left.V \in \mathfrak{A}\right\}$. In other words, $N(\boldsymbol{R})$ is the normalizer of $\boldsymbol{R}$ in $\mathfrak{A}$. Later, Anastasio defined an additional type, $M$-semiregular $(M=1,2,3, \ldots)$, which coincides with the semiregular type when $M=1$. Extending the notation $N(D)$ to any subring $D \subset \mathfrak{A}$, and letting $N^{j}(D)$ $=N\left[N^{j-1}(\boldsymbol{D})\right]$, we have a chain $\boldsymbol{R} \varsubsetneqq N(\boldsymbol{R}) \varsubsetneqq N^{2}(\boldsymbol{R}) \varsubsetneqq \cdots \varsubsetneqq N^{t}(\boldsymbol{R})=\mathfrak{A}$. We say that a maximal abelian subalgebra $\boldsymbol{R}$ is $M$-semiregular if $N^{k}(\boldsymbol{R})$ is not a factor for $k<M$, but $N^{M}(R)$ is a factor [1]. Anastasio constructed infinite sequences of nonisomorphic 2-semiregular and 3-semiregular subalgebras in a hyperfinite factor. (The 1-semiregular case had already been done [7].) In this paper we propose to show the existence of $M$-semiregular subalgebras for every positive integer $M \neq 1$.

We use the notation and results of [7]. Let $\mathfrak{M}_{p}$ be the full $2^{p}$ by $2^{p}$ matrix algebra over the complex numbers, and $\left\{{ }^{p} E_{i j}: i, j=0,1, \ldots, 2^{p}-1\right\}$ the matrix units which generate it. By letting ${ }^{p} E_{i j}={ }^{p+1} E_{2 i, 2 j}+{ }^{p+1} E_{2 i+1,2 j+1}$, we imbed $\mathfrak{M}_{p}$ in $\mathfrak{M}_{p+1}$. Then $\bigcup_{p=1}^{\infty} \mathfrak{M}_{p}=\mathfrak{M}$ is a ${ }^{*}$-algebra. The normalized matrix trace on $\mathfrak{M}$ makes it into a pre-Hilbert space $\mathfrak{S}$ : If $A, B \in \mathfrak{M}$, let $(A, B)=\operatorname{Tr}\left(B^{*} A\right)$, so that $(A, A)^{1 / 2}=\llbracket A \rrbracket$, the Hilbert space or metric norm of $A$. If $A$ is in $\mathfrak{M}$, then $A$ acting

Received by the editors November $21,1966$.

( ${ }^{1}$ This work was supported by the National Science Foundation under an Academic Year Extension of the Research Participation Program for College Teachers. 
by left multiplication is a bounded operator on $\mathfrak{S}$, so it can be extended to the Hilbert space closure $\mathscr{H}$. If $\mathfrak{A}$ is the weak closure of $\mathfrak{M}$, then it is well known that $\mathfrak{A}$ is a hyperfinite factor [2].

2. $M$-semiregular subalgebras. The following general construction leads to a large variety of maximal abelian subalgebras of $\mathfrak{A}$.

Definitions 2.1. Let $\left\{U_{t}: t=1,2, \ldots\right\}$ be a set of selfadjoint unitaries such that: (1) $U_{t} \in \mathfrak{M}_{t}$; (2) $U_{t}$ is zero except for 2 by 2 blocks along the main diagonal. Let $Y_{t}=U_{1} U_{2} \cdots U_{t}$, and for $A \in \mathfrak{A}$, define $A^{(t)}=Y_{t} A Y_{t}^{*}$ and $A^{[t]}=Y_{t}^{*} A Y_{t}$. For fixed $t$, the mappings $A \rightarrow A^{(t)}$ and $A \rightarrow A^{[t]}$ are *-automorphisms of $\mathfrak{A}$ and inverses of each other. Because of the form of $U_{t}$, the matrix unit ${ }^{p} E_{j j}$ commutes with $U_{t}$ for all $t>p$. Thus if $A$ is a diagonal matrix in $\mathfrak{M}_{p}, p \leqq t$, then $A^{(t)}=A^{(t+1)}$, and so $\lim _{t \rightarrow \infty} A^{(t)}=A^{(\infty)}$ exists in $\mathfrak{M}$, hence in $\mathfrak{A}$.

In general, for $A \in \mathfrak{A}$, the limit $A^{(\infty)}$ does not exist. The mapping $A \rightarrow A^{(\infty)}$ is thus an isomorphism of some proper subalgebra of $\mathfrak{A}$ into $\mathfrak{A}$. This subalgebra, the domain of the mapping, we call $\mathscr{D}$. If $E$ is the set of diagonal matrices, then $\boldsymbol{E} \subset \mathfrak{D}$, as seen above. The ring $\left(\boldsymbol{E}^{(\infty)}\right)^{-}$is the maximal abelian subalgebra $\boldsymbol{R}$ which we study in this paper. (Cf. [7, pp. 285-286], for the proof that $\boldsymbol{R}$ is maximal abelian.) In Lemma 2.2 we will show that $\boldsymbol{E}^{-} \subset \mathfrak{D}$, and that $\left(\boldsymbol{E}^{-}\right)^{(\infty)}=\left(\boldsymbol{E}^{(\infty)}\right)^{-}$or $\boldsymbol{R}$.

LEMMA 2.2. If $\boldsymbol{F}=\boldsymbol{E}^{-}$, then $\boldsymbol{F} \subset \mathfrak{D}$, and $\boldsymbol{F}^{(\infty)}=\left(\boldsymbol{E}^{(\infty)}\right)^{-}=\boldsymbol{R}$.

Proof. Suppose $A \in \boldsymbol{F}$. Then there is a sequence $A_{n} \in \boldsymbol{E} \cap \mathfrak{M}_{n}, A_{n} \rightarrow A$, with $A_{n}^{(\infty)} \in \mathfrak{M}$. Let $\varepsilon>0$ be given, and choose $n$ such that $\llbracket A_{n}-A \rrbracket<\varepsilon / 2$. Consider

$$
\begin{aligned}
\llbracket A^{(s)}-A^{(t)} \rrbracket & =\llbracket Y_{s} A Y_{s}^{*}-Y_{t} A Y_{t}^{*} \rrbracket \\
& \leqq \llbracket Y_{s} A Y_{s}^{*}-Y_{s} A_{n} Y_{s}^{*} \rrbracket+\llbracket Y_{s} A_{n} Y_{s}^{*}-Y_{t} A_{n} Y_{t}^{*} \rrbracket+\llbracket Y_{t} A_{n} Y_{t}^{*}-Y_{t} A Y_{t}^{*} \rrbracket .
\end{aligned}
$$

Choose $s, t$ such that both are greater than or equal to $n$. Then $Y_{s} A_{n} Y_{s}^{*}=A_{n}^{(s)}=A_{n}^{(n)}$ and $Y_{t} A_{n} Y_{t}^{*}=A_{n}^{(t)}=A_{n}^{(n)}$. Hence $\llbracket Y_{s} A_{n} Y_{s}^{*}-Y_{t} A_{n} Y_{t}^{*} \rrbracket=0$ if $s, t \geqq n$. Since $Y_{s}$ and $Y_{t}$ are unitary, the other two norms equal $\llbracket A-A_{n} \rrbracket$, and so the sum is less than $\varepsilon$. Therefore $A^{(t)}$ is Cauchy in the metric topology.

Now $A \in \mathfrak{A}$ and so $\|A\|<\infty$. Since $\left\|A^{(t)}\right\|=\|A\|, A^{(t)}$ is a bounded sequence. By [5, p. 723], $A^{(t)}$ is then Cauchy in the strong topology also, so its limit exists in $\mathfrak{A}$. Therefore $\boldsymbol{F} \subset \mathfrak{D}$.

We next show that $\boldsymbol{F}^{(\infty)}=\left(\boldsymbol{E}^{(\infty)}\right)^{-}$or $\boldsymbol{R}$. Let $A \in \boldsymbol{F}, A_{n} \in \boldsymbol{E} \cap \mathfrak{M}_{n}, A_{n} \rightarrow A$. Let $\varepsilon>0$ be given, and choose $n$ so that both $\llbracket A_{n}-A \rrbracket<\varepsilon / 2$ and $\llbracket A^{(n)}-A^{(\infty)} \rrbracket<\varepsilon / 2$. Then

$$
\begin{aligned}
\llbracket A_{n}^{(\infty)}-A^{(\infty)} \rrbracket & \leqq \llbracket A_{n}^{(\infty)}-A_{n}^{(n)} \rrbracket+\llbracket A_{n}^{(n)}-A^{(n)} \rrbracket+\llbracket A^{(n)}-A^{(\infty)} \rrbracket \\
& =\llbracket \llbracket A_{n}^{(n)}-A_{n}^{(n)} \rrbracket+\llbracket A_{n}-A \rrbracket+\llbracket A^{(n)}-A^{(\infty)} \rrbracket \\
& \leqq 0+\varepsilon / 2+\varepsilon / 2=\varepsilon .
\end{aligned}
$$

Therefore $A^{(\infty)} \in\left(E^{(\infty)}\right)^{-}$, and so $\boldsymbol{F}^{(\infty)} \subset \boldsymbol{R}$.

On the other hand, if $G \in R$, there is a sequence $A_{n} \in E \cap \mathfrak{M}_{n}, A_{n}^{(\infty)} \rightarrow G$, with $\left\|A_{n}^{(\infty)}\right\|=\left\|A_{n}^{(n)}\right\|=\left\|A_{n}\right\| \leqq\|G\|[4]$. Since $A_{n}^{(\infty)}$ is metrically Cauchy, so is $A_{n}$, which 
is also strongly Cauchy because of the bound on the norm. Hence $A_{n}$ has a limit $A \in F$. By another standard argument, if $\varepsilon>0$ be given, there exists $N$ such that $\llbracket Y_{t} A Y_{t}^{*}-G \rrbracket<\varepsilon$ when $t \geqq N$. Therefore $G=\lim _{t \rightarrow \infty} Y_{t} A Y_{t}^{*}$ and so $G \in F^{(\infty)}$. Hence $\boldsymbol{R} \subset \boldsymbol{F}^{(\infty)}$, and $\boldsymbol{F}^{(\infty)}=\boldsymbol{R}$.

REMARK. The normalizer of $\boldsymbol{R}$ in $\mathfrak{A}$ results in a similar way from the mapping $A \rightarrow A^{(\infty)}$. The subalgebra $r\left(\mathscr{C}_{0}^{D}\right)$, to be defined later, has the property that $\boldsymbol{E} \subset \boldsymbol{r}\left(\mathscr{C}_{0}^{D}\right) \subset \mathfrak{D}$, and $r\left(\mathscr{C}_{0}^{D}\right)^{(\infty)}=N(\boldsymbol{R})$. (It appears that $r\left(\mathscr{C}_{0}^{D}\right)=\mathfrak{D}$, but we do not need this fact and have not proved it.)

By means of various choices of the sequence $\left\{U_{i}\right\}$, in $\S 3$ we construct a maximal abelian subalgebra $\boldsymbol{R}_{n}$ for each $n=1,2,3, \ldots$, where $\boldsymbol{R}_{n}$ is $M$-semiregular, $M=n+1$. The chain $\boldsymbol{R}_{n} \varsubsetneqq N(\boldsymbol{R})=\boldsymbol{P}_{n} \varsubsetneqq N^{2}\left(\boldsymbol{R}_{n}\right) \varsubsetneqq \cdots \varsubsetneqq N^{n+1}\left(\boldsymbol{R}_{n}\right)=N^{M}\left(\boldsymbol{R}_{N}\right)=\mathfrak{2}$ is such that $N^{k}\left(\boldsymbol{R}_{n}\right)$ is not a factor for $k=1,2, \ldots, n<M$, while $N^{M}\left(\boldsymbol{R}_{n}\right)$ is the factor $\mathfrak{A}$.

Furthermore, the subalgebras $\boldsymbol{R}_{n}$ are not conjugate under any *-automorphism of $\mathfrak{A}$. The integer $n$ determines the number of normalizers between $\boldsymbol{R}_{n}$ and $\mathfrak{A}$ in the chain, and this is an automorphism invariant (cf. [7, pp. 282 and 305]).

Note. For convenience of notation, we often work with $N^{k}\left(\boldsymbol{P}_{n}\right)=N^{k+1}\left(\boldsymbol{R}_{n}\right)$, $k=0,1, \ldots, n$.

3. Detailed construction of $M$-semiregular subalgebras. In the construction of $M$-semiregular subalgebras, we use the following notations and definitions.

Definitions 3.1. We regard $n=1,2,3, \ldots$ as fixed, and let

$$
\Gamma=\{p: p=(3 c+1) n, c=0,1,2, \ldots\},
$$

an infinite set of positive integers. We define $\mathscr{C}_{n}=\left\{{ }^{p} E_{r s}: p \in \Gamma\right\}$. In the following paragraphs, we define a decomposition of $\mathscr{C}_{n}$ into $2^{n}$ disjoint subsets $K_{\gamma}$ $\left(0 \leqq \gamma \leqq 2^{n}-1\right)$, so that $\mathscr{C}_{n}=\bigcup_{\gamma} K_{\gamma}$.

Let $\mathscr{S}_{n}$ be the set of all $n$-tuples $\left(a_{1}, a_{2}, \ldots, a_{n}\right)$, where $a_{k}=0$ or 1 . This is a commutative group under the operation of coordinate-wise addition modulo 2 . If $\gamma=0,1, \ldots, 2^{n}-1$ and $\gamma=\sum_{j=1}^{n} a_{j} 2^{n-j}$, we identify it with its binary expansion $\left(a_{1}, a_{2}, \ldots, a_{n}\right)$, so that we can consider $\gamma$ as an element of $\mathscr{S S}_{n}$. The sum $\gamma_{1}+\gamma_{2}$ is then defined by addition in $\mathbb{S S}_{n}$.

We determine the set $K_{\gamma}$ in which ${ }^{p} E_{r s}$ is contained as follows: For any index $r$ $\left(0 \leqq r \leqq 2^{(3 c+1) n}-1\right)$, let $r=\sum_{k=0, k \neq 2}^{3 c} r_{k} 2^{k n}$. (Congruence is modulo 3 in this and in the following summations.) For $k \equiv 0$, we have $0 \leqq r_{k}<2^{n}$, and so $r_{k}=\sum_{j=1}^{n} k_{j} 2^{n-j}$ with $\left(k_{1}, \ldots, k_{n}\right) \in \mathbb{S}_{n}$. Designate this element of $\mathscr{S}_{n}$ by $\psi\left(r_{k}\right)$. For $k \equiv 1,0 \leqq r_{k}<2^{2 n}$, and we let $\sigma\left(r_{k}\right)=2\left(r_{k} \bmod 2^{n-1}\right)$, so that $\psi\left(\sigma\left(r_{k}\right)\right)$ is defined. Let

$$
\Delta(r)=\sum_{k \equiv 0 ; k=0}^{3 c} \psi\left(r_{k}\right)+\sum_{k \equiv 1 ; k=1}^{3 c-2} \psi\left(\sigma\left(r_{k}\right)\right),
$$

where the addition is coordinate-wise $(\bmod 2)$, so that $\Delta(r) \in \mathbb{S}_{n}$. Then $K_{y}=$ $\left\{{ }^{p} E_{r s}: \Delta(r)+\Delta(s)=\gamma\right\}$ and we say that $K_{\gamma}=K\left({ }^{p} E_{r s}\right)$. Since this is independent of $p$, we sometimes write $K_{\gamma}=K(r, s)$. 
DEFINITIONS 3.2. We also define the following sets of matrix units, again subsets of $\mathscr{C}_{n}: \mathscr{C}_{0}=\mathscr{N}_{0}=K_{0}$. For $j=1,2, \ldots, n, \mathscr{C}_{j}=\bigcup_{\gamma}\left\{K_{\gamma}: \gamma=\left(a_{1}, \ldots, a_{j}, 0,0, \ldots, 0\right)\right\}$ and $\mathscr{N}_{j}=\mathscr{C}_{j} \sim \mathscr{C}_{j-1}$. If we let

$$
\mathfrak{M}^{D}={ }^{1} E_{00} \mathfrak{M}^{1} E_{00}+{ }^{1} E_{11} \mathfrak{M}^{1} E_{11},
$$

then we define $\mathscr{C}_{j}^{D}=\mathscr{C}_{j} \cap \mathfrak{M}^{D}$ and $\mathscr{N}_{j}^{D}=\mathscr{N}_{j} \cap \mathfrak{M}^{D}$, while $\mathscr{C}_{j}^{\prime}=\mathscr{C}_{j} \sim \mathscr{C}_{j}^{D}$ and $\mathscr{N}_{j}^{\prime}=\mathscr{N}_{j} \sim \mathscr{N}_{j}^{D}$. We let $r\left(\mathscr{C}_{j}^{D}\right)$ be the ring generated by the matrix units in $\mathscr{C}_{j}^{D}$, while $R\left(\mathscr{C}_{j}^{D}\right)$ is the ring generated by $\left\{F: F=\left({ }^{p} E_{r s}\right)^{(p)}\right.$ with $\left.{ }^{p} E_{r s} \in \mathscr{C}_{j}^{D}\right\}$.

LEMMA 3.3. Suppose $p \in \Gamma,{ }^{p+3 n} E_{r s} \in K_{\gamma}$. Let $r=r^{\prime} 2^{3 n}+r_{1} 2^{n}+r_{0}$ and $s=s^{\prime} 2^{3 n}$ $+s_{1} 2^{n}+s_{0} \quad\left(0 \leqq r_{1}, s_{1}<2^{2 n}, 0 \leqq r_{0}, s_{0}<2^{n}\right)$. Then

$$
\gamma=\Delta(r)+\Delta(s)=\left(\Delta\left(r^{\prime}\right)+\Delta\left(s^{\prime}\right)\right)+\sigma+\left(\Delta\left(r_{0}\right)+\Delta\left(s_{0}\right)\right) \text {, }
$$

where $\sigma=\psi\left(\sigma\left(r_{1}\right)\right)+\psi\left(\sigma\left(s_{1}\right)\right)$.

Proof. This follows by computation from Definitions 3.1, since $\Delta(r)$ can be written as $\Delta\left(r^{\prime}\right)+\psi\left(\sigma\left(r_{1}\right)\right)+\psi\left(r_{0}\right)$, and the same for $\Delta(s)$.

Construction 3.4. In constructing the maximal abelian subalgebra $\boldsymbol{R}_{n}$ according to $\S 2.1$, the sequence $\left\{U_{t}: t=1,2,3, \ldots\right\}$ is to be as follows: Let

$$
B_{1}=\left[\begin{array}{cc}
2^{-1 / 2} & 2^{-1 / 2} \\
2^{-1 / 2} & -2^{-1 / 2}
\end{array}\right] \text {. }
$$

Let $B_{t}$ be in $\mathfrak{M}_{t}$, with all entries zero except for 2 by 2 blocks like $B_{1}$ along the main diagonal.

For $n>1, U_{t}=I$ if $t<n$. If $p \in \Gamma$ and if $\Delta(r)=\left(a_{1}, a_{2}, \ldots, a_{n}\right)$, define:

$$
\begin{aligned}
& { }^{p} E_{r r} U_{p+1}={ }^{p} E_{r r} \quad \text { if } a_{n}=0 \text {, } \\
& ={ }^{p} E_{r r} B_{p+1} \quad \text { if } a_{n}=1 \text {, } \\
& { }^{p} E_{r r} U_{p+n-j+1}={ }^{p} E_{r r} \quad \text { if } a_{j}=0 \text {, } \\
& ={ }^{p} E_{r r} B_{p+n-j+1} \quad \text { if } a_{j}=1, \\
& U_{p+n+i}=I \text {. } \\
& { }^{p} E_{r r} U_{p+n+2}={ }^{p} E_{r r} \quad \text { if } a_{2}=0 \text {, } \\
& ={ }^{p} E_{r r} B_{p+n+2} \quad \text { if } a_{2}=1 \text {, } \\
& { }^{p} E_{r r} U_{p+n+j}={ }^{p} E_{r r} \quad \text { if } a_{j}=0 \text {, } \\
& ={ }^{p} E_{r r} B_{p+n+j} \quad \text { if } a_{j}=1 \text {, } \\
& { }^{p} E_{r r} U_{p+2 n}={ }^{p} E_{r r} \quad \text { if } a_{n}=0, \\
& ={ }^{p} E_{r r} B_{p+2 n} \quad \text { if } a_{n}=1 \text {, } \\
& U_{p+2 n+1}=\cdots=U_{p+3 n-2}=I \text {, } \\
& { }^{1} E_{00} U_{p+3 n-1}={ }^{1} E_{00} \text {, } \\
& { }^{1} E_{11} U_{p+3 n-1}={ }^{1} E_{11} B_{p+3 n-1}, \\
& U_{p+3 n}=I \text {. }
\end{aligned}
$$


For $n=1, p \in \Gamma$, and if $\Delta(r)=\left(a_{1}\right)$, define:

$$
\begin{array}{rlrl}
{ }^{p} E_{r r} U_{p+1} & ={ }^{p} E_{r r} & & \text { if } a_{1}=0, \\
& ={ }^{p} E_{r r} B_{p+1} & & \text { if } a_{1}=1, \\
{ }^{1} E_{00} U_{p+2} & ={ }^{1} E_{00}, & & \\
{ }^{1} E_{11} U_{p+2} & ={ }^{1} E_{11} B_{p+2}, & & \\
U_{p+3} & =I . &
\end{array}
$$

REMARK. With this construction we aim to show that $N^{j+1}(R)=N^{j}(\boldsymbol{P})=R\left(\mathscr{C}_{j}^{D}\right)$ for $j=0,1, \ldots, n-1$, and that none of these is a factor. However,

$$
N^{n}(\boldsymbol{P})=\mathfrak{A}=R\left(\mathscr{C}_{n}^{D} \cup \mathscr{C}_{n}^{\prime}\right)
$$

(For $n=1$, the following three propositions hold with slight adaptations. Then nothing else is needed until Theorems 3.14 and 3.15.)

THEOREM 3.5. $N(\boldsymbol{R})=\boldsymbol{P}=R\left(\mathscr{C}_{0}^{D}\right)$.

Proof. If $p \in \Gamma,{ }^{p} E_{r s} \in \mathscr{C}_{0}^{D}$, then $\Delta(r)+\Delta(s)=(0,0, \ldots, 0)$. So computation with the definitions of $\$ 3.4$ shows that

$$
U_{p+3 n} \cdots U_{p+1}^{p} E_{r s} U_{p+1} \cdots U_{p+3 n}={ }^{p} E_{r s} .
$$

If $q \in \Gamma, q>p$, then $q=p+3 h n$ for some integer $h$. Since ${ }^{p} E_{r s}$ is a sum $\sum_{v}{ }^{q} E_{r_{v} s_{v}}$, with all terms of the sum in $\mathscr{C}_{0}^{D}$, we have

$$
U_{q} \cdots U_{p+1}^{p} E_{r s} U_{p+1} \cdots U_{q}={ }^{p} E_{r s} \in \mathscr{C}_{0}^{D} .
$$

But if ${ }^{p} E_{r s} \in \mathscr{N}_{j}(j \geqq 1)$, then

$$
U_{p+n-j+1} \cdots{ }^{p} E_{r s} \cdots U_{p+n-j+1}={ }^{p} E_{r s} B_{p+n-j+1} .
$$

Also, if ${ }^{p} E_{r s} \in \mathscr{C}_{0}^{\prime}$,

$$
U_{p+3 n-1} \cdots{ }^{p} E_{r s} \cdots U_{p+3 n-1}={ }^{p} E_{r s} B_{p+3 n-1} .
$$

Hence our construction satisfies the conditions of [7, $\$ 4.1]$, with $\mathscr{C}_{0}^{D}$ taking the place of $K_{0}$. Also, $d \leqq 3 n-1$ is surely sufficient. Thus we can apply [7, Lemma 4.3] in order to conclude that any unitary $V$ leaving $R$ invariant is the metric limit of a sequence $V_{m}$ in $\mathfrak{M}$ such that if $V_{m} \in \mathfrak{M}_{p}(p \in \Gamma)$, then $V_{m}^{[p]}=\sum \alpha_{c d}{ }^{p} E_{c d}$ with ${ }^{p} E_{c d} \in \mathscr{C}_{0}^{D}$. So if $V \in N(\boldsymbol{R})$, then $V \in R\left(\mathscr{C}_{0}^{D}\right)$, and we have $N(\boldsymbol{R}) \subset R\left(\mathscr{C}_{0}^{D}\right)$.

On the other hand, consider a unitary $V$ in $\mathfrak{M}_{p}(p \in \Gamma)$ such that $V^{[p]}=\sum \pm{ }^{p} E_{r s}$ with ${ }^{p} E_{r s} \in \mathscr{C}_{0}^{D}$ and signs arbitrary. It is straightforward to show that $V$ leaves $\boldsymbol{R}$ invariant. Since the collection of all unitaries of this type is sufficient to generate $R\left(\mathscr{C}_{0}^{D}\right)$, we have $R\left(\mathscr{C}_{0}^{D}\right) \subset N(\boldsymbol{R})$.

Therefore $N(\boldsymbol{R})=\boldsymbol{P}=\boldsymbol{R}\left(\mathscr{C}_{0}^{D}\right)$.

REMARK. The preceding proof also implies that $r\left(\mathscr{C}_{0}^{D}\right)$ is in $\mathscr{D}$ and that $R\left(\mathscr{C}_{0}^{D}\right)=r\left(\mathscr{C}_{0}^{D}\right)^{(\infty)}$. For if $F=\sum \alpha_{r s}{ }^{p} E_{r s}$ with ${ }^{p} E_{r s} \in \mathscr{C}_{0}^{D}$, then $F^{(p)}=F^{(p+h)}$ for any $h>0$. Hence $\lim _{p \rightarrow \infty} F^{(p)}=F^{(\infty)}$ exists and $F \in \mathscr{D}$. Using this information about $F \in r\left(\mathscr{C}_{0}^{D}\right) \cap \mathfrak{M}$, Lemma 2.2 and its proof can be rephrased to show that $r\left(\mathscr{C}_{0}^{D}\right) \subset \mathfrak{D}$, 
and that $R\left(\mathscr{C}_{0}^{D}\right)$, which is defined as the closure of $\left[r\left(\mathscr{C}_{0}^{D}\right) \cap \mathfrak{M}\right]^{(\infty)}$, can also be regarded simply as $r\left(\mathscr{C}_{0}^{D}\right)^{(\infty)}$.

LEMMA 3.6. Let $p \in \Gamma, A^{[p]}=\sum \alpha_{c d}^{p} E_{c d}$ with ${ }^{p} E_{c d}$ in $\mathscr{N}_{j}^{D}(0 \leqq j \leqq n), \mathscr{C}_{n-1}^{\prime}$, or $\mathscr{N}_{n}^{\prime}$. Then if $q \in \Gamma, q>p, A^{[q]}=\sum \beta_{r s}^{q} E_{r s}$ with ${ }^{q} E_{r s}$ also in $\mathscr{N}_{j}^{D}, \mathscr{C}_{n-1}^{\prime}$, or $\mathscr{N}_{n}^{\prime}$ respectively.

Proof. The case $\mathscr{N}_{0}^{D}$ has already been dealt with, since $\mathscr{N}_{0}^{D}=\mathscr{C}_{0}^{D}$.

We first consider $q=p+3 n$. Then $A^{[q]}=U_{p+3 n} \cdots A^{[p]} \cdots U_{p+3 n}$, and because of linearity it is sufficient to consider one term of $A^{[p]}$, say ${ }^{p} E_{c d}$.

If $1 \leqq j \leqq n$ and ${ }^{p} E_{c d} \in \mathscr{N}_{j}^{D}$, then Definition 3.4 shows that

$$
U_{p+3 n} \cdots{ }^{p} E_{c d} \cdots U_{p+3 n}=\sum \delta_{r s}^{p+3 n} E_{r s}
$$

is in $\mathfrak{M}_{p+n+j}$. Consider one term ${ }^{p+3 n} E_{r s}$. With $r=c \cdot 2^{3 n}+r_{1} 2^{n}+r_{0}$ and $s=d \cdot 2^{3 n}+s_{1} 2^{n}+s_{0}$, we thus have $r_{0}=s_{0}$ and $r_{1} \equiv s_{1}\left(\bmod 2^{n-j}\right)$. So $\sigma\left(r_{1}\right)=\sigma\left(s_{1}\right)$ $\left(\bmod 2^{n-j+1}\right)$; and therefore $\psi\left(\sigma\left(r_{1}\right)\right)+\psi\left(\sigma\left(s_{1}\right)\right)=\left(a_{1}, \ldots, a_{j-1}, 0,0, \ldots\right)$, while $\psi\left(r_{0}\right)+\psi\left(s_{0}\right)=(0,0,0, \ldots)$. Hence, applying Lemma $3.3,{ }^{p+3 n} E_{r s} \in \mathscr{N}_{j}$ as was ${ }^{p} E_{c d}$. Now the action of the unitaries $U_{t}$ surely preserves $\mathscr{C}_{n}^{D}$, and therefore ${ }^{p+3 n} E_{r s}$ is in $\mathscr{N}_{j}^{D}$.

Next suppose ${ }^{p} E_{c d} \in \mathscr{C}_{n-1}^{\prime}$ and consider $U_{p+3 n} \cdots{ }^{p} E_{c d} \cdots U_{p+3 n}$. The product is in $\mathfrak{M}_{p+3 n-1}$, by Definition 3.4 , so one term ${ }^{p+3 n} E_{r s}$ has $r=c \cdot 2^{3 n}+r_{1} 2^{n}+r_{0}$, $s=d \cdot 2^{3 n}+s_{1} 2^{n}+s_{0}$ with $r_{0} \equiv s_{0}(\bmod 2)$. Thus $\psi\left(r_{0}\right)+\psi\left(s_{0}\right)=\left(\ldots, a_{n-1}, 0\right)$, and we can have ${ }^{p+3 n} E_{r s} \in \mathscr{N}_{n}$ if and only if $\psi\left(\sigma\left(r_{1}\right)\right)+\psi\left(\sigma\left(s_{1}\right)\right)=\left(\ldots, a_{n-1}, 1\right)$. But by definition, $\sigma\left(r_{1}\right) \equiv 0(\bmod 2)$, so this cannot happen. As before, the action of the $U_{t}^{\prime}$ 's preserves $\mathscr{C}_{n}^{\prime}$. Therefore ${ }^{p+3 n} E_{r s}$ is in $\mathscr{C}_{n-1}^{\prime}$.

If ${ }^{p} E_{c d} \in \mathscr{N}_{n}^{\prime}$, then this time the computations of the preceding paragraph lead to the conclusion that the terms of $U_{p+3 n} \cdots{ }^{p} E_{c d} \cdots U_{p+3 n}$ are in $\mathcal{N}_{n}^{\prime}$. (Here $\left.\psi\left(\sigma\left(r_{1}\right)\right)+\psi\left(\sigma\left(s_{1}\right)\right)=\left(\ldots, a_{n-1}, 0\right).\right)$

If $q \in \Gamma, q>p$, then $q=p+3 h n$ for some integer $h$, and the desired result follows by induction.

LeMMA 3.7. For $j=1,2, \ldots, n, R\left(\mathscr{C}_{j-1}^{D}\right) \varsubsetneqq R\left(\mathscr{C}_{j}^{D}\right) \varsubsetneqq R\left(\mathscr{C}_{n}\right)$.

Proof. The inclusions are trivial and we need only show that they are proper inclusions.

Let $F$ be a matrix unit in $\mathscr{N}_{j}^{D}$ (resp. $\left.\mathscr{C}_{n}^{\prime}\right)$, so that $F \in \mathfrak{M}_{p}(p \in \Gamma)$ and $F^{[p]}={ }^{p} E_{a b}$ in $\mathscr{N}_{j}^{D}\left(\mathscr{C}_{n}^{\prime}\right)$. Suppose that $F$ is also in $R\left(\mathscr{C}_{j-1}^{D}\right)\left(R\left(\mathscr{C}_{n}^{D}\right)\right)$. Then there is a sequence $F_{m} \in \mathfrak{M}$ converging strongly to $F$, such that if $F_{m} \in \mathfrak{M}_{q}(q \in \Gamma), F_{m}^{[q]}=\sum \beta_{c d}{ }^{q} E_{c d}$ with ${ }^{q} E_{c d} \in \mathscr{C}_{j-1}^{D}\left(\mathscr{C}_{n}^{D}\right)$. Choose $F_{m}$ such that $\llbracket F_{m}-F \rrbracket<1 / 2^{p}$ and choose $q \in \Gamma$ such that $F_{m}, F \in \mathfrak{M}_{q}$. Then by Lemma 3.6, $F^{[q]}=\sum \alpha_{a b}{ }^{q} E_{a b}$ with ${ }^{q} E_{a b} \in \mathscr{N}_{j}^{D}\left(\mathscr{C}_{n}^{\prime}\right)$.

Case 1. $F \in \mathscr{N}_{j}^{D}$. Since $\left(F_{m}^{[q]}, F^{[q]}\right)=\left(F_{m}, F\right)=0$, we have $1 / 2^{2 p}>\llbracket F_{m}-F \rrbracket^{2}=\llbracket F_{m} \rrbracket^{2}$ $+\llbracket F \rrbracket^{2}>1 / 2^{p}$, a contradiction. Therefore $F \notin R\left(\mathscr{C}_{j-1}^{D}\right)$.

Case 2. $F \in \mathscr{C}_{n}^{\prime}$. Here

$$
\begin{aligned}
\left(F_{m}^{[q]}, F^{[q]}\right) & =\left({ }^{1} E_{i \mathfrak{i}} F_{m}^{[q]}{ }^{1} E_{i \mathfrak{i}}+{ }^{1} E_{j j} F_{m}^{[q]}{ }^{1} E_{j j},{ }^{1} E_{i \mathfrak{i}} F^{[q]}{ }^{1} E_{j j}\right) \\
& =0 \quad \text { where } i, j=0 \text { or } 1, i \neq j .
\end{aligned}
$$

So again $1 / 2^{2 p}>\left[F_{m}-F \rrbracket^{2}>1 / 2^{p}\right.$, a contradiction, and therefore $F \notin R\left(\mathscr{C}_{n}^{D}\right)$. 
Definition 3.8. We define the following projections in $\mathscr{C}_{0}^{D}$ : For $k=2, \ldots, n$ and $s=0,1, \ldots, 2^{p}-1$, let $P_{k}(s)$ be the operator such that $P_{k}(s)^{[p+3 n]}=\sum_{h}{ }^{p+3 n} E_{8^{n}+h, 8^{n}+h}$, where $s^{\prime \prime}=2^{3 n} s, h \equiv 0\left(\bmod 2^{2 n-k+1}\right)$ and $0 \leqq h \leqq 2^{3 n}-1$. Let $P^{\prime}(s)$ be the operator such that $P^{\prime}(s)^{[p+3 n]}=\sum_{h}{ }^{p+3 n} E_{s^{\prime \prime}+h, s^{\prime \prime}+h}$, where $s^{\prime \prime}=2^{3 n} s, h \equiv 0\left(\bmod 2^{2}\right)$ and $0 \leqq h \leqq 2^{3 n}-1$.

Lemma 3.9. Suppose $W \in \mathfrak{M}_{p}(p \in \Gamma)$ is such that $W^{[p]}=V^{[p]}+X^{[p]}$, with $V^{[p]}=\sum \beta_{r s}{ }^{p} E_{r s}\left({ }^{p} E_{r s} \in \mathscr{C}_{n}^{D}\right)$ and $X^{[p]}=\sum \alpha_{r s}{ }^{p} E_{r s}\left({ }^{p} E_{r s} \in \mathscr{C}_{n}^{\prime}\right)$. Let ${ }^{p} E_{r t}$ be a fixed matrix unit in $\mathscr{C}_{n}^{D}$ with $K(r, t)=K_{\gamma}$. Then

$$
\begin{gathered}
{ }^{p} E_{r r}\left[U_{p+3 n} \cdots W^{[p]} \cdots U_{p+3 n}\right] \sum_{s=0}^{2^{p}-1} P^{\prime}(s)^{[p+3 n]}\left[U_{p+3 n} \cdots W^{*[p]} \cdots U_{p+3 n}\right]^{p} E_{t t} \\
=A(r, t)^{[p+3 n]}+Q(r, t)^{[p+3 n]}
\end{gathered}
$$

where $(A, Q)=0$ and

$$
Q^{[p+3 n]}=\sum_{s=0}^{2 p-1} \alpha_{r s} \bar{\alpha}_{t s} C(\gamma)^{p+3 n} E_{a b}
$$

with ${ }^{p+3 n} E_{a b}$ in $\mathscr{N}_{n-1}^{D}$ or $\mathscr{N}_{n}^{D}, C(\gamma)$ a nonzero integer.

Proof. The following statements are verified by calculations similar to those of [7, pp. 295-301].

Suppose $K\left({ }^{p} E_{r s}\right)=K_{\alpha}$ and $K\left({ }^{p} E_{s t}\right)=K_{\beta}$, with both matrix units in $\mathscr{C}_{n}^{\prime}, \alpha+\beta=\gamma$. If $\alpha=\left(a_{1}, a_{2}, \ldots, a_{n}\right)$ and $\beta=\left(b_{1}, b_{2}, \ldots, b_{n}\right)$, define $\omega_{1}=\omega(\alpha)=2\left(\sum_{i=2}^{n} a_{i}\right)+a_{1}+1$, $\omega_{2}=\omega(\beta)$, and $\mu(\alpha, \beta)=2\left(\sum_{i=2}^{n} a_{i} b_{i}\right)+a_{1} b_{1}$. Then the nonzero entries of the product $U_{p+3 n} \cdots{ }^{p} E_{r s} \cdots U_{p+3 n}$ have numerical value $\pm\left(2^{-1 / 2}\right)^{\omega_{1}}$, and similarly for ${ }^{p} E_{s t}$. Let $r_{0}=2^{3 n-2} r, s_{0}=2^{3 n-2} s, t_{0}=2^{3 n-2} t$. Then $2^{\mu}$ is the number of distinct $\delta$ 's such that

and

$$
{ }^{p+3 n-2} E_{r_{0} r_{0}}\left[U_{p+3 n} \cdots{ }^{p} E_{r s} \cdots U_{p+3 n}\right]^{p+3 n-2} E_{s_{0}+\delta, s_{0}+\delta}
$$

$$
{ }^{p+3 n-2} E_{s_{0} s_{0}}\left[U_{p+3 n} \cdots{ }^{p} E_{r s} \cdots U_{p+3 n}\right]^{p+3 n-2} E_{t_{0}+\delta, t_{0}+\delta}
$$

are both nonzero.

Using the preceding, a matrix calculation shows that

$$
\left[U_{p+3 n} \cdots{ }^{p} E_{r s} \cdots U_{p+3 n}\right] P^{\prime}(s)^{[p+3 n]}\left[U_{p+3 n} \cdots{ }^{p} E_{r s} \cdots U_{p+3 n}\right]
$$

has a term of the form $C(\gamma)^{p+3 n} E_{r^{n}, t^{\prime \prime}+2}$ and a term of the form $C(\gamma)^{p+3 n} E_{r^{\prime \prime}+2, t^{\prime \prime}+2}$, where $r^{\prime \prime}=2^{3 n} r, t^{\prime \prime}=2^{3 n} t$, and $C(\gamma)=2^{\mu}\left(2^{-1 / 2}\right)^{\omega_{1}+\omega_{2}}$. It is straightforward to show that $C(\gamma)$ depends only on $\gamma$ and on the fact that ${ }^{p} E_{r s}$ and ${ }^{p} E_{s t}$ are in $\mathscr{C}_{n}^{\prime}$. By Lemma 3.3 , if $K_{\gamma} \subset \mathscr{C}_{n-2}$, then $K\left(r^{\prime \prime}, t^{\prime \prime}+2\right)$ is in $\mathscr{N}_{n-1}$. If $K_{\gamma} \subset \mathscr{N}_{n-1}$ or $\mathscr{N}_{n}$, then so is $K\left(r^{\prime \prime}+2, t^{\prime \prime}+2\right)$. Also, since ${ }^{p} E_{r t} \in \mathscr{C}_{n}^{D}$, so are these matrix units.

Now the product $(* *)$ of the lemma equals

$$
\sum_{s=0}^{2^{p}-1}\left[U_{p+3 n} \cdots \delta_{r s}^{p} E_{r s} \cdots U_{p+3 n}\right] P^{\prime}(s)^{[p+3 n]}\left[U_{p+3 n} \cdots \delta_{t s}^{p} E_{s t} \cdots U_{p+3 n}\right]
$$


Suppose $K_{y} \subset \mathscr{C}_{n-2}$ and $s$ such that ${ }^{p} E_{r s}$ and ${ }^{p} E_{s t}$ are both in $\mathscr{C}_{n}^{\prime}$. The summand corresponding to this $s$ includes the term $\alpha_{r s} \bar{a}_{t s} C(\gamma)^{p+3 n} E_{r^{\prime \prime}, t^{\prime \prime}+2}$, which is in $\mathscr{N}_{n-1}$. Considering the summands corresponding to other $s$, we could not have one matrix unit in $\mathscr{C}_{n}^{\prime}$, the other in $\mathscr{C}_{n}^{D}$, since ${ }^{p} E_{r t} \in \mathscr{C}_{n}^{D}$. But if both are in $\mathscr{C}_{n}^{D}$, then the product is in $\mathfrak{M}_{p+2 n}$, so there is no element in position $\left(r^{\prime \prime}, t^{\prime \prime}+2\right)$.

Suppose $K_{\gamma} \subset \mathscr{N}_{n}$ or $\mathscr{N}_{n-1}$. If $s$ is such that ${ }^{p} E_{r s}$ and ${ }^{p} E_{s t}$ are both in $\mathscr{C}_{n}^{\prime}$, then the summand includes the term $\alpha_{r s} \bar{a}_{t s} C(\gamma)^{p+3 n} E_{r^{\prime \prime}+2, t^{\prime \prime}+2}$, which is in the same class as $K_{y}$. Again, if $s$ is such that both matrix units are in $\mathscr{C}_{n}^{D}$ there is no element in position $\left(r^{\prime \prime}+2, t^{\prime \prime}+2\right)$.

So if we let $Q$ be as stated in the lemma, with $(a, b)=\left(r^{\prime \prime}, t^{\prime \prime}+2\right)$ or $\left(r^{\prime \prime}+2, t^{\prime \prime}+2\right)$ according to $K_{r}$, then $(A, Q)=0$ and ${ }^{p+3 n} E_{a b} \in \mathscr{N}_{n-1}^{D}$ or $\mathscr{N}_{n}^{D}$.

LEMma 3.10. Suppose $W \in \mathfrak{M}_{p}(p \in \Gamma)$ is such that $W^{[p]}=V^{[p]}+X^{[p]}$, with $V^{[p]}=\sum \beta_{r s}{ }^{p} E_{r s}\left({ }^{p} E_{r s} \in \mathscr{C}_{k-1}^{D}\right)$ and $X^{[p]}=\sum \alpha_{r s}{ }^{p} E_{r s}\left({ }^{p} E_{r s} \in \mathscr{N}_{k}^{D}\right)$. Let ${ }^{p} E_{r t}$ be a fixed matrix unit in $\mathscr{C}_{k-1}^{D}$ with $K(r, t)=K_{r}$. Then

$$
\begin{gathered}
{ }^{p} E_{r r}\left[U_{p+3 n} \cdots W^{[p]} \cdots U_{p+3 n}\right] \sum_{s=0}^{2^{p}-1} P_{k}(s)^{[p+3 n]}\left[U_{p+3 n} \cdots W^{*[p]} \cdots U_{p+3 n}\right]^{p} E_{t t} \\
=A(r, t)^{[p+3 n]}+Q(r, t)^{[p+3 n]}
\end{gathered}
$$

where

$$
Q^{[p+3 n]}=\sum_{s=0}^{2^{p}-1} \alpha_{r s} \bar{a}_{t s} D_{k}(\gamma)^{p+3 n} E_{a b}
$$

with ${ }^{p+3 n} E_{a b}$ in $\mathscr{N}_{k-1}^{D}, D_{k}(\gamma)$ a nonzero integer.

Proof. The proof is like that of the preceding lemma, with the following changes: $\omega_{1}=\omega(\alpha)=2\left(\sum_{i=2}^{n} a_{i}\right)+a_{1}$ (and a similar change in $\left.\omega_{2}\right), \mu(\alpha, \beta)=2\left(\sum_{i=2}^{k-1} a_{i} b_{i}\right)+a_{k} b_{k}$ $+a_{1} b_{1}, r_{0}=2^{n+k-1} r, s_{0}=2^{n+k-1} s, t_{0}=2^{n+k-1} t$. Then $2^{\mu}$ is the number of distinct $\delta$ 's such that

$$
{ }^{p+n+k-1} E_{r_{0} r_{0}}\left[U_{p+3 n} \cdots{ }^{p} E_{r s} \cdots U_{p+3 n}\right]^{p+n+k-1} E_{s_{0}+\delta, s_{0}+\delta}
$$

and

$$
{ }^{p+n+k-1} E_{s_{0} s_{0}}\left[U_{p+3 n} \cdots{ }^{p} E_{s t} \cdots U_{p+3 n}\right]^{p+n+k-1} E_{t_{0}+\delta, t_{0}+\delta}
$$

are both nonzero. The expression

$$
\left[U_{p+3 n} \cdots{ }^{p} E_{r s} \cdots U_{p+3 n}\right] P_{k}(s)^{[p+3 n]}\left[U_{p+3 n} \cdots{ }^{p} E_{s t} \cdots U_{p+3 n}\right]
$$

has a term of the form $D_{k}(\gamma)^{p+3 n} E_{r^{n}, t^{n}+\pi}$ and a term of the form $D_{k}(\gamma)^{p+3 n} E_{r^{n}+\pi, t^{*}+\pi}$ where $r^{\prime \prime}=2^{3 n} r, t^{\prime \prime}=2^{3 n} t, \pi=2^{2 n-k}$, and $D_{k}(\gamma)=2^{\mu}\left(2^{-1 / 2}\right)^{\omega_{1}+\omega_{2}}$. Here $D_{k}(\gamma)$ depends only on $\gamma$ and on $k$. By Lemma 3.3, if $K_{\gamma} \subset \mathscr{C}_{k-2}$, then $K\left(r^{\prime \prime}, t^{\prime \prime}+\pi\right)$ is in $\mathscr{N}_{k-1}$; if $K_{r} \subset \mathscr{N}_{k-1}$, then $K\left(r^{\prime \prime}+\pi, t^{\prime \prime}+\pi\right)$ is in $\mathscr{N}_{k-1}$.

It can be verified, as in the preceding lemma, that $(A, Q)=0$ if we take $Q$ as stated, with $(a, b)=\left(r^{\prime \prime}, t^{\prime \prime}+\pi\right)$ or $\left(r^{\prime \prime}+\pi, t^{\prime \prime}+\pi\right)$ according to $K_{r}$. 
LEMMA 3.11. If the results of Lemmas 3.9 and 3.10 hold for $q=p+3 n$, then they hold for any $q=p+3 h n$ (i.e., $q \in \Gamma$ ). Also,

$$
\llbracket Q \rrbracket^{2} \geqq\left|\sum_{s=0}^{2 p-1} \alpha_{r s} \bar{a}_{t s}\right|^{2} / 2^{p+5 n} .
$$

Proof. We first obtain bounds for $C(\gamma)$ and $D_{k}(\gamma)$. In both cases, we have $\mu \geqq 0$ and $\omega_{1}+\omega_{2} \leqq 2(2 n-1)+2=4 n$. Hence $C(\gamma)$ or $D_{k}(\gamma)=2^{\mu}\left(2^{-1 / 2}\right)^{\omega_{1}+\omega_{2}} \geqq\left(2^{-1 / 2}\right)^{4 n}$ $=1 / 2^{2 n}$.

$$
\begin{aligned}
\llbracket Q^{[p+3 n]} \rrbracket^{2} & \geqq|C(\gamma)|^{2}\left|\sum \alpha_{r s} \bar{\alpha}_{t s}\right|^{2} / 2^{p+3 n} \\
& \geqq\left|\sum \alpha_{r s} \bar{\alpha}_{t s}\right|^{2} / 2^{p+5 n},
\end{aligned}
$$

and similarly in the case of $D_{k}(\gamma)$.

Now the unitaries $U_{p+3 n+1}, \ldots, U_{p+3 h n}$ preserve the orthogonality of $A$ and $Q$ and the norm of $Q$. Also, by Lemma 3.6, matrix units in $\mathscr{N}_{j}^{D}(j=1,2, \ldots, n)$ are left in that class under the action of the unitaries $U_{t}$.

Lemma 3.12. For $j=1,2, \ldots, n-1$, let $\mathscr{V}_{j}=\left\{V: V\left[R\left(\mathscr{C}_{j-1}^{D}\right)\right] V^{*}=R\left(\mathscr{C}_{j-1}^{D}\right), \quad V\right.$ unitary, $V \in \mathfrak{A}\}$. If $V \in \mathscr{V}_{j}$, then there is a sequence $V_{m} \in \mathfrak{M}$ converging metrically to $V$ such that if $V_{m} \in \mathfrak{M}_{p}(p \in \Gamma), V_{m}^{[p]}=\sum \beta_{r s}{ }^{p} E_{r s}$ with ${ }^{p} E_{r s}$ in $\mathscr{C}_{j}^{D}$. Thus, $N\left(R\left(\mathscr{C}_{j-1}^{D}\right)\right)$ $\subset R\left(\mathscr{C}_{j}^{D}\right)$.

Proof. (i) Since $V \in \mathfrak{A},\|V\| \leqq 1$, there is a sequence $W_{m} \in \mathfrak{M},\left\|W_{m}\right\| \leqq 1$, converging strongly and metrically to $V$ [4]. If $W_{m} \in \mathfrak{M}_{p}$, let $W_{m}^{[p]}=V_{m}^{[p]}+X_{m}^{[p]}$, where $V_{m}^{[p]}=\sum \beta_{r s}^{p} E_{r s}\left({ }^{p} E_{r s} \in \mathscr{C}_{n}^{D}\right)$ and $X_{m}^{[p]}=\sum \alpha_{r s}{ }^{p} E_{r s}\left({ }^{p} E_{r s} \in \mathscr{C}_{n}^{\prime}\right)$. Because of the orthogonality of $V_{m}$ and $X_{m}, X_{m}$ itself is Cauchy in the metric topology. Now $\left\|W_{m}\right\| \leqq 1$ implies $\left\|V_{m}\right\| \leqq 1$ because of the definition of $\mathscr{C}_{n}^{D}$. Since $X_{m}=W_{m}-V_{m}$, we have $\left\|X_{m}\right\| \leqq 2$, and so $X_{m}$ is also Cauchy in the strong topology [5, p. 723]. Let $X_{m} \rightarrow X \in \mathfrak{A}$. Suppose $\lim _{m} \llbracket X_{m} \rrbracket \neq 0$; then $\lim _{m} \llbracket X_{m} X_{m}^{*} \rrbracket \neq 0$ also. Hence $\llbracket X_{m} X_{m}^{*} \rrbracket^{2}$ $>2^{5 n} \varepsilon^{2}$ for all $m$ and some $\varepsilon>0$. (Recall that $n$ is fixed and related only to $\boldsymbol{R}_{n} \boldsymbol{R}_{n}$.)

Choose $W_{m}$ so that $\llbracket W_{m}-V \rrbracket<\varepsilon / 4$. Suppose $W_{m} \in \mathfrak{M}_{p}$. Then

$$
\llbracket X_{m}^{[p]} X_{m}^{[p] *} \rrbracket^{2}=\left(1 / 2^{p}\right) \sum\left|\sum_{s=0}^{2^{p}-1} \alpha_{r s} \bar{\alpha}_{t s}\right|^{2}>2^{5 n} \varepsilon^{2} .
$$

(The outer summation is over pairs $(r, t)$ such that ${ }^{p} E_{r t} \in \mathscr{C}_{n}^{D}$, since ${ }^{p} E_{r s},{ }^{p} E_{s t} \in \mathscr{C}_{n}^{\prime}$.) Fix $p$ from here on.

Consider $\sum_{s=0}^{2^{p}-1} P^{\prime}(s)^{[p+3 n]}$, which has its matrix units in $\mathscr{C}_{0}^{D}$. Then $\sum_{s} P^{\prime}(s)$ is in $R\left(\mathscr{C}_{j-1}^{D}\right)$ for any $j \geqq 1$, and if $V \in \mathscr{V}_{j}, V\left(\sum_{s} P^{\prime}(s)\right) V^{*}=T \in R\left(\mathscr{C}_{j-1}^{D}\right)$. So there exists a sequence $T_{v} \in \mathfrak{M}$, $\left[T_{v}-T \rrbracket \rightarrow 0\right.$, and $T_{v} \in \mathfrak{M}_{q}(q \in \Gamma)$ implies $T_{v}^{[q]}=\sum \eta_{i h}{ }^{q} E_{i h}$ with ${ }^{q} E_{i h}$ in $\mathscr{C}_{j-1}^{D}$. Choose $T_{v}$ such that $\llbracket V\left(\sum_{s} P^{\prime}(s)\right) V^{*}-T_{v} \rrbracket<\varepsilon / 2$. Since $\sum_{s} P^{\prime}(s)$ is a projection, of norm at most one,

$$
\llbracket W_{m}\left(\sum_{s} P^{\prime}(s)\right) W_{m}^{*}-V\left(\sum_{s} P^{\prime}(s)\right) V^{*} \rrbracket<\varepsilon / 2,
$$


and thus it follows that

$$
\llbracket W_{m}\left(\sum_{s} P^{\prime}(s)\right) W_{m}^{*}-T_{v} \rrbracket<\varepsilon .
$$

On the other hand, we can apply Lemmas 3.9 and 3.11 with $W_{m}$ replacing $W$. Take $q$ to be such that $q \in \Gamma, q \geqq p+3 n$, and $T_{v} \in \mathfrak{M}_{q}$. Since $Q^{[q]}=\sum \lambda_{c d}{ }^{q} E_{c d}$ $\left({ }^{q} E_{c d} \in \mathscr{N}_{n-1}^{D}\right.$ or $\left.\mathscr{N}_{n}^{D}\right)$ and $T_{v}^{[q]}=\sum \eta_{i h}{ }^{q} E_{i h}\left({ }^{q} E_{i h} \in \mathscr{C}_{j-1}^{D}\right.$, where $\left.j-1<n-1\right)$, we have $\left(T_{v}^{[q]}, Q^{[q]}\right)=0$ also. Therefore

$$
\begin{aligned}
{\left[{ }^{p} E_{r r} W_{m}^{[q]} \sum\right.} & P^{\prime}(s)^{[q]} W_{m}^{*[q] p} E_{t t}-{ }^{p} E_{r r} T_{v}^{[q] p} E_{t t} \rrbracket^{2} \\
& =\llbracket A(r, t)^{[q]}+Q(r, t)^{[q]}-{ }^{p} E_{r r} T_{v}^{[q] p} E_{t t} \rrbracket^{2} \\
& \geqq \llbracket Q(r, t)^{[q]} \rrbracket^{2} \geqq\left|\sum_{s} \alpha_{r s} \bar{\alpha}_{t s}\right|^{2} / 2^{p+5 n} .
\end{aligned}
$$

Finally, we have:

$$
\begin{aligned}
\varepsilon^{2} & \geqq \sum_{(r, t)} \llbracket\left[{ }^{p} E_{r r}\left(W_{m}^{[q]} \sum_{s} P^{\prime}(s) W_{m}^{*[q]}-T_{v}^{[q]}\right){ }^{p} E_{t t}\right]^{2}, \quad{ }^{p} E_{r t} \in \mathscr{C}_{n}^{D} \\
& \geqq \sum_{(r, t)}\left|\sum_{s} \alpha_{r s} \bar{\alpha}_{t s}\right|^{2} / 2^{p+5 n}>\varepsilon^{2},
\end{aligned}
$$

which is a contradiction.

Therefore $\lim _{l} \llbracket X_{l} \rrbracket=0$ and so $\lim _{l} \llbracket V_{l}-V \rrbracket=0$, where $\left\|V_{l}\right\| \leqq 1$ and $V_{l} \in \mathfrak{M}_{z}$ $(z \in \Gamma)$ implies $V_{l}^{[z]}=\sum \beta_{r s}{ }^{z} E_{r s}$ with ${ }^{z} E_{r s} \in \mathscr{C}_{n}^{D}$.

(ii) To show: Suppose $j<k \leqq n$ and suppose there exists $W_{m} \in \mathfrak{M}$ such that $\left\|W_{m}\right\| \leqq 1, \lim _{m} \llbracket W_{m}-V \rrbracket=0$, and $W_{m} \in \mathfrak{M}_{p}$ implies $W_{m}^{[p]}=\sum \delta_{r s}^{p} E_{r s}$ with ${ }^{p} E_{r s}$ in $\mathscr{C}_{k}^{D}$. Then there exists $V_{m}$ with the same properties except that $V_{m}^{[p]}=\sum \beta_{r s}{ }^{p} E_{r s}$ with ${ }^{p} E_{r s}$ in $\mathscr{C}_{k-1}^{D}$.

We let the assumed $W_{m}^{[p]}=V_{m}^{[p]}+X_{m}^{[p]}$, where the matrix units of the two summands are in $\mathscr{C}_{k-1}^{D}$ and $\mathscr{N}_{k}^{D}$ respectively. The argument proceeds much as in part (i), with $\sum_{s} P_{k}(s)$ replacing $\sum_{s} P^{\prime}(s)$, so that Lemmas 3.10 and 3.11 apply. Since $V\left(\sum P_{k}(s)\right) V^{*}=T$ in $R\left(\mathscr{C}_{j-1}^{D}\right)$ and since $j-1<k-1$, the desired orthogonality holds between $Q$ (in $\mathscr{N}_{k-1}^{D}$ ) and $T_{v}$ (the sequence of matrices converging to $T$ ). We are led to conclude that $\lim _{m} \llbracket X_{m} \rrbracket=0$, and that $V$ is the metric limit of $V_{m}$.

Since we can extend this as far as $k=j+1$ by a finite induction process, the lemma is proved.

THEOREM 3.13. For $j=1,2, \ldots, n-1$, if $R\left(\mathscr{V}_{j}\right)$ is the ring generated by $\mathscr{V}_{j}$ as defined in Lemma 3.12, then $R\left(\mathscr{V}_{j}\right)=R\left(\mathscr{C}_{j}^{D}\right)$. Thus, $N\left(R\left(\mathscr{C}_{j-1}^{D}\right)\right)=R\left(\mathscr{C}_{j}^{D}\right)$.

Proof. By Lemma 3.12, $R\left(\mathscr{V}_{j}\right) \subset R\left(\mathscr{C}_{j}^{D}\right)$.

For the reverse inclusion, take $T \in R\left(\mathscr{C}_{j-1}^{D}\right)$. Let $V_{1}^{[p]}=\sum \pm{ }^{p} E_{r s}$ with ${ }^{p} E_{r s}$ in $\mathscr{C}_{j-1}^{D}$ and signs arbitrary. Then $V_{1} T V_{1}^{*}$ is in $R\left(\mathscr{C}_{j-1}^{D}\right)$ since all three operators are. 
Next let $V_{2}^{[p]}=\sum \pm{ }^{p} E_{r s}$ with ${ }^{p} E_{r s}$ in $\mathscr{N}_{j}^{D}$. Take a sequence $T_{m} \in \mathfrak{M}, T_{m} \rightarrow T$, and if $T \in \mathfrak{M}_{q}, T_{m}^{[q]}=\sum \beta_{c d}{ }^{q} E_{c d}$ with ${ }^{q} E_{c d}$ in $\mathscr{C}_{j-1}^{D}$. If $z=\max [p, q]$, then

$$
V_{2}^{[q]} T_{m}^{[q]} V_{2}^{*[q]}=\left[\sum \delta_{r s}^{z} E_{r s}\right]\left[\sum \beta_{c d}^{\prime z} E_{c d}\right]\left[\sum \delta_{r s}{ }^{z} E_{r s}\right],
$$

where the matrix units of the first sum are in $\mathscr{N}_{j}^{D}$, those of the second in $\mathscr{C}_{j-1}^{D}$, and those of the third in $\mathscr{N}_{j}^{D}$, by Lemma 3.6. Calculating by means of $\S 3.1$, we see that each matrix unit of this product is in $\mathscr{C}_{j-1}^{D}$. Hence $V_{2} T_{m} V_{2}^{*}$ is in $R\left(\mathscr{C}_{j-1}^{D}\right)$, and so is its strong limit $V_{2} T V_{2}^{*}$.

But all unitaries of the form $V_{1}$ or $V_{2}$ are sufficient to generate $R\left(\mathscr{C}_{j}^{D}\right)$. Therefore $R\left(\mathscr{C}_{j}^{D}\right) \subset R\left(\mathscr{V}_{j}\right)$, and hence $R\left(\mathscr{V}_{j}\right)=R\left(\mathscr{C}_{j}^{D}\right)$.

THEOREM 3.14. If $\mathscr{V}_{n}=\left\{V: V\left[R\left(\mathscr{C}_{n-1}^{D}\right)\right] V^{*}=R\left(\mathscr{C}_{n-1}^{D}\right), V\right.$ unitary, $\left.V \in \mathfrak{A}\right\}$, then $R\left(\mathscr{V}_{n}\right)=R\left(\mathscr{C}_{n}\right)=\mathfrak{A}$. Thus, $N\left(R\left(\mathscr{C}_{n-1}^{D}\right)\right)=\mathfrak{A}$.

Proof. Obviously $R\left(\mathscr{V}_{n}\right) \subset R\left(\mathscr{C}_{n}\right)$.

For the reverse inclusion, let $T$ be in $R\left(\mathscr{C}_{n-1}^{D}\right)$. Consider in turn four types of unitaries $V_{i}^{[p]}=\sum \pm{ }^{p} E_{r s}(i=1,2,3,4$ and signs arbitrary). For $i=1$, the matrix units are to be in $\mathscr{C}_{n-1}^{D}$; for $i=2$, in $\mathscr{N}_{n}^{D}$; for $i=3$, in $\mathscr{C}_{n-1}^{\prime}$; for $i=4$, in $\mathscr{N}_{n}^{\prime}$. By Lemma 3.6, these classes are preserved under the unitaries $U_{t}$. So calculations like those in the proof of Theorem 3.13 show that $V_{i} T V_{i}^{*}$ is in $R\left(\mathscr{C}_{n-1}^{D}\right)$ for $i=1,2,3,4$.

But all unitaries of these types are sufficient to generate $R\left(\mathscr{C}_{n}\right)$, or $\mathfrak{A}$. Therefore $R\left(\mathscr{C}_{n}\right) \subset R\left(\mathscr{V}_{n}\right)$, and $R\left(\mathscr{V}_{n}\right)=\mathfrak{A}$.

REMARK. Theorems 3.13 and 3.14, together with Theorem 3.5 and Lemma 3.7, show that for each $\boldsymbol{R}_{n}, n=1,2,3, \ldots$, we have $\boldsymbol{R}_{n} \varsubsetneqq N\left(\boldsymbol{R}_{n}\right) \varsubsetneqq \cdots \varsubsetneqq N^{n+1}\left(\boldsymbol{R}_{n}\right)=\mathfrak{A}$. In order to prove that $\boldsymbol{R}_{n}$ is $M$-semiregular $(n+1=M)$, we need only show that $N\left(\boldsymbol{R}_{n}\right), N^{2}\left(\boldsymbol{R}_{n}\right), \ldots, N^{n}\left(\boldsymbol{R}_{n}\right)$ are not factors. $\left(N^{n+1}\left(\boldsymbol{R}_{n}\right)=N^{M}\left(\boldsymbol{R}_{n}\right)\right.$ is the factor $\left.\mathfrak{A}.\right)$

THEOREM 3.15. For $k=1,2, \ldots, n, N^{k}\left(\boldsymbol{R}_{n}\right)$ is not a factor.

Proof. If $k \neq n, N^{k}\left(\boldsymbol{R}_{n}\right)=N^{k-1}\left(\boldsymbol{P}_{n}\right)=R\left(\mathscr{V}_{k-1}\right)=R\left(\mathscr{C}_{k-1}^{D}\right)$. Consider the projection ${ }^{1} E_{00}={ }^{1} E_{00}^{(\infty)} \in \boldsymbol{R}_{n} \subset N^{k}\left(\boldsymbol{R}_{n}\right)$. If $A$ is any operator in $N^{k}\left(\boldsymbol{R}_{n}\right)$, there is a sequence $A_{m} \rightarrow A$ such that if $A_{m} \in \mathfrak{M}_{p}, A_{m}^{[p]}=\sum \alpha_{r s}^{p} E_{r s}$ with ${ }^{p} E_{r s} \in \mathscr{C}_{k-1}^{D}$. Then

$$
\begin{aligned}
\left({ }^{1} E_{00} A_{m}{ }^{1} E_{00}\right)^{[p]} & ={ }^{1} E_{00} A_{m}^{[p] 1} E_{00}=\sum \alpha_{r s}{ }^{1} E_{00}{ }^{p} E_{r s}{ }^{1} E_{00} \\
& =\sum \alpha_{r s}{ }^{p} E_{r s} \quad \text { (by definition of } \mathscr{C}_{k-1}^{D} \text { ) } \\
& =A_{m}^{[p]} .
\end{aligned}
$$

Thus ${ }^{1} E_{00} A_{m}{ }^{1} E_{00}=A_{m}$, and taking strong limits, ${ }^{1} E_{00} A{ }^{1} E_{00}=A$.

Therefore ${ }^{1} E_{00}$ commutes with $N^{k}\left(\boldsymbol{R}_{n}\right),{ }^{1} E_{00} \neq \alpha I,{ }^{1} E_{00} \in N^{k}\left(\boldsymbol{R}_{n}\right)$, and so $N^{k}\left(\boldsymbol{R}_{n}\right)$ is not a factor.

\section{BIBLIOGRAPHY}

1. S. Anastasio, Maximal abelian subalgebras in hyperfinite factors, Amer. J. Math. 87 (1965), 955-971.

2. J. Dixmier, Les algèbres d'opérateurs dans l'espace Hilbertien, Gauthier-Villars, Paris, 1957.

3. - Sous-anneaux abéliens maximaux dans les facteurs de type fini, Ann. of Math. (2) 59 (1954), 279-286. 
4. I. Kaplansky, $A$ theorem on rings of operators, Pacific J. Math. 1 (1951), 227-232.

5. F. J. Murray and J. von Neumann, On rings of operators. IV, Ann. of Math. (2) 44 (1943), 716-808.

6. J. von Neumann, On rings of operators. Reduction theory, Ann. of Math. (2) 50 (1949), 401-485.

7. Sister R. J. Tauer, Maximal abelian subalgebras in finite factors of type II, Trans. Amer. Math. Soc. 114 (1965), 281-308.

The College of St. Catherine,

St. Paul, Minnesota 